EPJ Web of Conferences 72, 00006 (2014)

DOI: 10.1051 /epjconf / 20147200006

C Owned by the authors, published by EDP Sciences, 2014

\title{
Kaon Physics at KLOE/KLOE-2: recent results
}

\author{
Antonio Di Domenico ${ }^{1,2, a}$ \\ on behalf of the KLOE-2 collaboration ${ }^{b}$ \\ ${ }^{1}$ Dipartimento di Fisica, Sapienza Università di Roma, Italy \\ ${ }^{2}$ INFN sezione di Roma, Italy
}

\begin{abstract}
The results of the most recent analyses using kaon data collected by the KLOE experiment at the Frascati $\phi$-factory, DA $\Phi \mathrm{NE}$, are presented: a new improved measurement of $\mathrm{BR}\left(\mathrm{K}^{+} \rightarrow \pi^{+} \pi^{-} \pi^{+}(\gamma)\right)$, a new improved search of the rare $\mathrm{CP}$ violating decay $\mathrm{K}_{\mathrm{S}} \rightarrow 3 \pi^{0}$, and new stringent limits on the CPT violating parameters $\Delta a_{\mu}$ for neutral kaons in the context of the Standard Model Extension framework.
\end{abstract}

\section{Introduction}

DAФNE, the Frascati $\phi$-factory is an $e^{+} e^{-}$collider working at a center of mass energy of $\sqrt{s} \sim 1020 \mathrm{MeV}$, corresponding to the peak of the $\phi$ resonance. The $\phi$-meson production cross section is $\sim 3 \mu \mathrm{b}$, and its decay yields $\sim 1.410^{6} \mathrm{~K}^{+} \mathrm{K}^{-}$pairs and $\sim 10^{6} \mathrm{~K}^{0} \overline{\mathrm{K}}^{0}$ pairs per $\mathrm{pb}^{-1}$ of integrated luminosity.

The KLOE experiment at DA $\Phi$ NE completed its first data taking campaign in March 2006 with a total integrated luminosity of $\sim 2.5 \mathrm{fb}^{-1}$, corresponding to a production of $\sim 7.5 \times 10^{9} \phi$-mesons. The KLOE detector is a $4 \pi$ detector setup, which is able to measure both charged and neutral particles. It consists of a large volume drift chamber (DC) [1], which provides excellent momentum and vertex reconstruction for charged particles, and a barrel shaped electromagnetic calorimeter (EMC) with two end-caps [2], made from lead and scintillating fibers, which surrounds the drift chamber. The energy deposits of charged and neutral particles in the calorimeter are measured with very good time resolution, which allows for the identification of charged particles based on their time of flight. Drift chamber and calorimeter are enclosed in a superconducting solenoid, providing an axial $0.52 \mathrm{~T}$ magnetic field.

\footnotetext{
a-mail: antonio.didomenico@roma1.infn.it

${ }^{\mathrm{b}}$ KLOE-2 collaboration: D. Babusci, I. Balwierz-Pytko, G. Bencivenni, C. Bloise, F. Bossi, P. Branchini, A. Budano, L. Caldeira Balkeståhl, G. Capon, F. Ceradini, P. Ciambrone, F. Curciarello, E. Czerwiński, E. Danè, V. De Leo, E. De Lucia, G. De Robertis, A. De Santis, P. De Simone, A. Di Cicco, A. Di Domenico, C. Di Donato, R. Di Salvo, D. Domenici, O. Erriquez, G. Fanizzi, A. Fantini, G. Felici, S. Fiore, P. Franzini, A. Gajos, P. Gauzzi, G. Giardina, S. Giovannella, E. Graziani, F. Happacher, L. Heijkenskjöld, B. Höistad, M. Jacewicz, T. Johansson, K. Kacprzak, D. Kamińska, A. Kupsc, J. Lee-Franzini, F. Loddo, S. Loffredo, G. Mandaglio, M. Martemianov, M. Martini, M. Mascolo, R. Messi, S. Miscetti, G. Morello, D. Moricciani, P. Moskal, F. Nguyen, A. Palladino, A. Passeri, V. Patera, I. Prado Longhi, A. Ranieri, P. Santangelo, I. Sarra, M. Schioppa, B. Sciascia, M. Silarski, C. Taccini, L. Tortora, G. Venanzoni, W. Wiślicki, M. Wolke, J. Zdebik.
}

\section{Absolute $\mathrm{K}^{+} \rightarrow \pi^{+} \pi^{-} \pi^{+}(\gamma)$ branching ratio measurement}

The measurement of the BR of the $\mathrm{K}^{+} \rightarrow \pi^{+} \pi^{-} \pi^{+}(\gamma)$ decay would complete the KLOE program of precise and fully inclusive kaon dominant BR's measurements. An indirect measurement is obtained by fitting the KLOE $\mathrm{K}^{ \pm}$lifetime and the other dominant BR's measurements, where the sum of the BR's is constrained to one [3]: $\quad \operatorname{BR}\left(\mathrm{K}^{+} \rightarrow \pi^{+} \pi^{-} \pi^{+}(\gamma)\right)=(5.68 \pm 0.22) \%$. Also the Flavianet working group obtained a value using all the available measurements on charged kaons [4]: $\mathrm{BR}\left(\mathrm{K}^{+} \rightarrow \pi^{+} \pi^{-} \pi^{+}(\gamma)\right)=(5.73 \pm 0.16) \%$ The most recent direct measurement, $\mathrm{BR}\left(\mathrm{K}^{ \pm} \rightarrow \pi^{ \pm} \pi^{+} \pi^{-}\right)=(5.56 \pm 0.20) \%$ [5], dates back to more than 30 years ago. Here, a new improved result obtained with $174 \mathrm{pb}^{-1}$ of the KLOE data is reported.

The selection of the $\mathrm{K}^{+}$beam is done reconstructing the two body decays $\mathrm{K}^{-} \rightarrow \pi^{-} \pi^{0}$ and $\mathrm{K}^{-} \rightarrow \mu^{-} \bar{v}(v)$, which are about $85 \%$ of the $\mathrm{K}^{-}$decays. These decays are identified as two clear peaks in the momentum of the charged secondary tracks in the kaon rest frame. To minimize the impact of the trigger efficiency on the signal side, only events given by the $\mathrm{K}_{\mu 2}$ tags are chosen as a normalization sample, which provide by themselves the calorimetric trigger of the event; this request reduces the normalization sample by $\sim 35 \%$.

The track of the tagging kaon, $\mathrm{K}^{-}$, is backward extrapolated to the interaction point, then the kinematics of the $\phi \rightarrow \mathrm{K}^{+} \mathrm{K}^{-}$decay defines direction and momentum of the signal kaon, $\mathrm{K}^{+}$. The decay products of the kaons have low momentum, less than $200 \mathrm{MeV} / \mathrm{c}$, and curl up in the KLOE magnetic field; this increases the probability to reconstruct bad quality tracks and fake vertices. Good quality reconstructed events are selected by requiring kaon decays before the inner wall of the DC (transverse radius $\rho_{x y}<25 \mathrm{~cm}$, geometrical acceptance $\sim 26 \%$ ) in order to reduce the maximum number of tracks inside the DC to 
three. Specifically, at least two reconstructed tracks in the DC (pion candidates) are required, then if their backward extrapolations form a vertex with the extrapolated path of the signal kaon before the inner wall of the DC, signal events are counted in the missing mass spectrum of the third pion.

A vertex is selected by cutting on the distance of closest approach between each extrapolated (pion) track and the signal kaon path, DCA $<3 \mathrm{~cm}$, and on the distance of closest approach between the two selected (pion) tracks, $\mathrm{DCA}_{t t}<3 \mathrm{~cm}$. To remove the background due to the two-body decays, each selected track is required to have a momentum in the kaon rest frame less than $190 \mathrm{MeV} / \mathrm{c}$. After this selection, a residual background, mainly due to badly reconstructed tracks, is removed requiring $\left|\cos \left(\theta_{12}\right)\right|$ $<0.90$, with $\theta_{12}$ the opening angle between the two selected tracks.

Fig.1 (left) shows the comparison between data (blue) and MC (red) of the missing mass spectrum, the MC background contribution (black) is superimposed; the signal over background ratio within the missing mass window $\left(10000 \mathrm{MeV}^{2}<m_{\text {miss }}^{2}<30000 \mathrm{MeV}^{2}\right)$ is $\mathrm{S} / \mathrm{B} \simeq 88.40$. In order to extract the number of signal events, the missing mass spectrum is fitted using signal and background distribution shapes as given by the MC. Fig.1 (right) shows the result of the fit (purple) superimposed to the data points (black), and the bottom plot shows the corresponding normalized residuals.

The selection efficiency $\epsilon_{\text {sel }}=0.0793 \pm 0.0003$ is evaluated from MC, applying corrections evaluated using a control sample of $\mathrm{K}^{+} \rightarrow \pi^{-} \mathrm{X}$ decays to account for data-MC differences in track reconstruction.

Finally the KLOE preliminary result is:

$$
\begin{array}{r}
\operatorname{BR}\left(\mathrm{K}^{+} \rightarrow \pi^{+} \pi^{+} \pi^{-}(\gamma)\right)= \\
=0.05526 \pm 0.00035_{\text {stat }} \pm 0.00036_{\text {syst }} .
\end{array}
$$

The main contributions to the systematic error are due to the cuts on the tracking variables, DCA and $\mathrm{DCA}_{t t}$.

\section{Neutral $\mathrm{K}$ mesons at KLOE}

In the $\mathrm{K}^{0}-\overline{\mathrm{K}}^{0}$ system the physical states $\left|\mathrm{K}_{\mathrm{S}}\right\rangle,\left|\mathrm{K}_{\mathrm{L}}\right\rangle$, i.e. the states with definite masses $m_{S, L}$ and lifetimes $\tau_{S, L}$ which evolve as a function of the kaon proper time $t$ as pure exponentials

$$
\begin{aligned}
& \left|\mathrm{K}_{\mathrm{S}}(t)\right\rangle=e^{-i \lambda_{S} t}\left|\mathrm{~K}_{\mathrm{S}}\right\rangle, \\
& \left|\mathrm{K}_{\mathrm{L}}(t)\right\rangle=e^{-i \lambda_{L} t}\left|\mathrm{~K}_{\mathrm{L}}\right\rangle,
\end{aligned}
$$

with $\lambda_{S, L}=m_{S, L}-i \Gamma_{S, L} / 2$, and $\Gamma_{S, L}=\left(\tau_{S, L}\right)^{-1}$, can be expressed in terms of the $\mathrm{CP}$ eigenstates

as:

$$
\begin{aligned}
& \left|\mathrm{K}_{1}\right\rangle=\frac{1}{\sqrt{2}}\left[\left|\mathrm{~K}^{0}\right\rangle+\left|\overline{\mathrm{K}}^{0}\right\rangle\right](\mathrm{CP}=+1) \\
& \left|\mathrm{K}_{2}\right\rangle=\frac{1}{\sqrt{2}}\left[\left|\mathrm{~K}^{0}\right\rangle-\left|\overline{\mathrm{K}}^{0}\right\rangle\right](\mathrm{CP}=-1)
\end{aligned}
$$

$$
\begin{aligned}
& \left|\mathrm{K}_{\mathrm{S}}\right\rangle=\frac{1}{\sqrt{\left(1+\left|\epsilon_{S}\right|^{2}\right)}}\left[\left|\mathrm{K}_{1}\right\rangle+\epsilon_{S}\left|\mathrm{~K}_{2}\right\rangle\right], \\
& \left|\mathrm{K}_{\mathrm{L}}\right\rangle=\frac{1}{\sqrt{\left(1+\left|\epsilon_{L}\right|^{2}\right)}}\left[\left|\mathrm{K}_{2}\right\rangle+\epsilon_{L}\left|\mathrm{~K}_{1}\right\rangle\right],
\end{aligned}
$$

with $\epsilon_{S}$ and $\epsilon_{L}$ two small complex parameters describing the $\mathrm{CP}$ impurity in the physical states. One can equivalently define $\epsilon \equiv\left(\epsilon_{S}+\epsilon_{L}\right) / 2$, and $\delta \equiv\left(\epsilon_{S}-\epsilon_{L}\right) / 2$; adopting a suitable phase convention (e.g. the Wu-Yang phase convention [6]) $\epsilon \neq 0$ implies T violation, $\delta \neq 0$ implies CPT violation, while $\delta \neq 0$ or $\epsilon \neq 0$ implies CP violation.

At a $\phi$-factory neutral kaons are produced in entangled pairs with the $\phi$-meson quantum numbers $J^{P C}=1^{--}$:

$$
\begin{aligned}
|i\rangle & =\frac{1}{\sqrt{2}}\left\{\left|\mathrm{~K}^{0}\right\rangle\left|\overline{\mathrm{K}}^{0}\right\rangle-\left|\overline{\mathrm{K}}^{0}\right\rangle\left|\mathrm{K}^{0}\right\rangle\right\} \\
& =\frac{\mathcal{N}}{\sqrt{2}}\left\{\left|\mathrm{~K}_{\mathrm{S}}\right\rangle\left|\mathrm{K}_{\mathrm{L}}\right\rangle-\left|\mathrm{K}_{\mathrm{L}}\right\rangle\left|\mathrm{K}_{\mathrm{S}}\right\rangle\right\},
\end{aligned}
$$

where $\mathcal{N}=\sqrt{\left(1+\left|\epsilon_{S}\right|^{2}\right)\left(1+\left|\epsilon_{L}\right|^{2}\right)} /\left(1-\epsilon_{S} \epsilon_{L}\right) \simeq 1$ is a normalization factor.

The observable quantity is the double differential decay rate of the state $|i\rangle$ into decay products $f_{1}$ and $f_{2}$ at proper times $t_{1}$ and $t_{2}$, respectively. After integration on $\left(t_{1}+t_{2}\right)$ at fixed time difference $\Delta t=t_{1}-t_{2}$, the decay intensity can be written as follows [7]:

$$
\begin{gathered}
I\left(f_{1}, f_{2} ; \Delta t \geq 0\right)=C_{12}\left\{\left|\eta_{1}\right|^{2} e^{-\Gamma_{L} \Delta t}+\left|\eta_{2}\right|^{2} e^{-\Gamma_{S} \Delta t}\right. \\
\left.-2\left|\eta_{1}\right|\left|\eta_{2}\right| e^{-\frac{\left.\Gamma_{S}+\Gamma_{L}\right)}{2} \Delta t} \cos \left[\Delta m \Delta t+\phi_{2}-\phi_{1}\right]\right\},
\end{gathered}
$$

with $\Delta m=m_{L}-m_{S}$,

$$
\begin{aligned}
\eta_{i} & \equiv\left|\eta_{i}\right| e^{i \phi_{i}}=\frac{\left\langle f_{i}|T| \mathrm{K}_{\mathrm{L}}\right\rangle}{\left\langle f_{i}|T| \mathrm{K}_{\mathrm{S}}\right\rangle}, \\
C_{12} & =\frac{|\mathcal{N}|^{2}}{2\left(\Gamma_{S}+\Gamma_{L}\right)}\left|\left\langle f_{1}|T| \mathrm{K}_{\mathrm{S}}\right\rangle\left\langle f_{2}|T| \mathrm{K}_{\mathrm{S}}\right\rangle\right|^{2} .
\end{aligned}
$$

This expression is valid for $\Delta t \geq 0$, while for $\Delta t<0$ the substitutions $\Delta t \rightarrow|\Delta t|$ and $1 \leftrightarrow 2$ have to be applied.

Due to the huge difference in the lifetimes of the physical states $\left(\tau_{L} \gg \tau_{S}\right)$, for $t_{1} \gg t_{2}, \tau_{S}$ (or $\left.t_{2} \gg t_{1}, \tau_{S}\right)$ the decay intensity in eq.(5) behaves like the initial state were an incoherent mixture of states $\left|\mathrm{K}_{\mathrm{S}}\right\rangle\left|\mathrm{K}_{\mathrm{L}}\right\rangle$ and $\left|\mathrm{K}_{\mathrm{L}}\right\rangle\left|\mathrm{K}_{\mathrm{S}}\right\rangle$. Hence the detection of a kaon at large times tags a $\mathrm{K}_{\mathrm{S}}$ in the opposite direction. This is a unique feature at a $\phi$-factory, not possible at fixed target experiments, that can be exploited to select pure $\mathrm{K}_{\mathrm{S}}$ beams.

\section{Search for $C P$ violation in $K_{S}$ decay}

The decay $\mathrm{K}_{\mathrm{S}} \rightarrow 3 \pi^{0}$ violates $\mathrm{CP}$ invariance. The parameter $\eta_{000}$, defined as the ratio of $\mathrm{K}_{\mathrm{S}}$ to $\mathrm{K}_{\mathrm{L}}$ decay amplitudes, can be written as:

$$
\eta_{000}=\frac{\left\langle 3 \pi^{0}|T| \mathrm{K}_{\mathrm{S}}\right\rangle}{\left\langle 3 \pi^{0}|T| \mathrm{K}_{\mathrm{L}}\right\rangle}=\epsilon_{S}+\epsilon_{000}^{\prime},
$$

where $\epsilon_{S}$ quantifies the $\mathrm{K}_{\mathrm{S}} \mathrm{CP}$ impurity and $\epsilon_{000}^{\prime}$ is due to a direct CP-violating term. Since we expect $\epsilon_{000}^{\prime} \ll \epsilon_{S}$ (at lowest order in Chiral Perturbation Theory one has [8, 9]: $\epsilon_{000}^{\prime}=-2 \epsilon^{\prime}$, with $\epsilon^{\prime}$ the direct $\mathrm{CP}$ violation parameter in $\pi \pi$ decays), it follows that $\eta_{000} \sim \epsilon_{S}$, and therefore in the Standard Model one has (assuming CPT invariance, i.e. $\left.\epsilon_{S}=\epsilon\right) \operatorname{BR}\left(\mathrm{K}_{\mathrm{S}} \rightarrow 3 \pi^{0}\right) \sim 1.9 \times 10^{-9}$ to an accuracy of a 

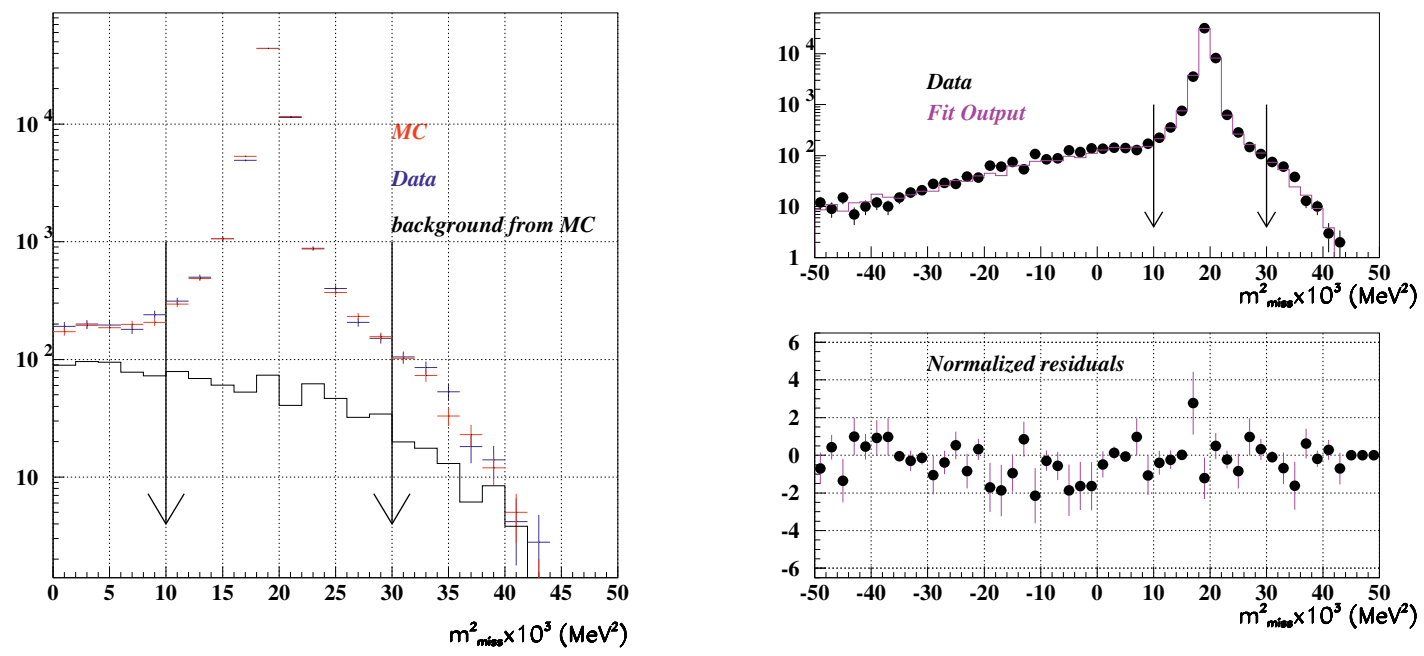

Figure 1. Right: missing mass spectrum for data (blue points), MC signal plus background (red points), and MC background only (black histogram); the arrows correspond to the missing mass window. Left: fit of the missing mass spectrum (purple) superimposed to data points (black). The bottom plot shows the normalized residuals of the fit.

few $\%$, making the direct observation of this decay quite a challenge.

The best upper limit on $\mathrm{BR}\left(\mathrm{K}_{\mathrm{S}} \rightarrow 3 \pi^{0}\right)$ comes from the analysis of $450 \mathrm{pb}^{-1}$ data collected by the KLOE experiment in years 2001-2002 [10]: $\mathrm{BR}\left(\mathrm{K}_{\mathrm{S}} \rightarrow 3 \pi^{0}\right)<$ $1.2 \times 10^{-7}$ at $90 \%$ C.L. Here, the result of a new improved analysis using $1.7 \mathrm{fb}^{-1}$ of data collected by KLOE in years 2004-2005 is reported.

The $\mathrm{K}_{\mathrm{S}}$ mesons are identified with high efficiency $(\sim 34 \%)$ via detection of $\mathrm{K}_{\mathrm{L}}$ mesons which cross the drift chamber without decaying and then interact with the electromagnetic calorimeter. The $\mathrm{K}_{\mathrm{S}} 4$-momentum is then determined using the measured position of the $\mathrm{K}_{\mathrm{L}}$ meson and the known momentum of the $\phi$ meson, which is estimated as an average of the momentum distribution measured using large angle Bhabha scattering events. The search for the $\mathrm{K}_{\mathrm{S}} \rightarrow 3 \pi^{0} \rightarrow 6 \gamma$ decay is then carried out by selecting events with six photons with momenta reconstructed using time and energy measured by the electromagnetic calorimeter. Background originates mainly from the $\mathrm{K}_{\mathrm{S}} \rightarrow 2 \pi^{0}$ events with two spurious clusters from fragmentation of the electromagnetic showers (splitting) or accidental activity, or from false $\mathrm{K}_{\mathrm{L}}$ identification for $\phi \rightarrow \mathrm{K}_{\mathrm{S}} \mathrm{K}_{\mathrm{L}} \rightarrow \pi^{+} \pi^{-}, 3 \pi^{0}$ events. In the latter case charged pions from $\mathrm{K}_{\mathrm{S}}$ decays interact in the DAФNE low- $\beta$ insertion quadrupoles, ultimately simulating the $\mathrm{K}_{\mathrm{L}}$ interaction in the calorimeter, while $\mathrm{K}_{\mathrm{L}}$ decays close to the IP producing six photons. To suppress this kind of background, events with charged particles coming from the vicinity of the interaction region are firstly rejected. Moreover a cut on the reconstructed velocity and energy of the tagging $\mathrm{K}_{\mathrm{L}}$ meson is also applied. In the next stage of the analysis a kinematic fit with 11 constraints is performed: energy and momentum conservation, the kaon mass and the velocity of the six photons. Cutting on the $\chi^{2}$ of the fit considerably reduces the background from bad quality reconstructed events with a very good signal efficiency. In order to reject events with split and accidental clusters the correlation between two $\chi^{2}$-like discriminating variables $\chi_{2 \pi}^{2}$ and $\chi_{3 \pi}^{2}$ is exploited. $\chi_{2 \pi}^{2}$ is calculated by an algorithm selecting four out of six clusters best satisfying the kinematic constraints of the two-body decay, therefore it verifies the $\mathrm{K}_{\mathrm{S}} \rightarrow 2 \pi^{0} \rightarrow 4 \gamma$ hypothesis. The pairing of clusters is based on the requirement $m_{\gamma \gamma}=m_{\pi^{0}}$ and on the opening angle of the reconstructed pions trajectories in the $\mathrm{K}_{\mathrm{S}}$ center of mass frame. Moreover the consistency of the energy and momentum conservation in the $\phi \rightarrow \mathrm{K}_{\mathrm{S}} \mathrm{K}_{\mathrm{L}}, \mathrm{K}_{\mathrm{S}} \rightarrow 2 \pi^{0}$ decay hypothesis is required; $\chi_{3 \pi}^{2}$ instead verifies the signal hypothesis by looking at the reconstructed masses of three pions. For every choice of cluster pairs, the quadratic sum of the residuals between the nominal $\pi^{0}$ mass and the invariant masses of three photon pairs are calculated. In order to improve the quality of the photon selection using $\chi_{2 \pi}^{2}$, a cut on the variable $\Delta E=\left(m_{\phi} / 2-\sum E_{\gamma_{i}}\right) / \sigma_{E}$ is applied where $\gamma_{i}$ stands for the $i$-th photon from four chosen in the $\chi_{2 \pi}^{2}$ estimator and $\sigma_{E}$ is the appropriate resolution. For $\mathrm{K}_{\mathrm{S}} \rightarrow 2 \pi^{0}$ decays plus two background clusters $\Delta E \sim 0$ is expected, while for $\mathrm{K}_{\mathrm{S}} \rightarrow 3 \pi^{0} \Delta E \sim m_{\pi^{0}} / \sigma_{E}$. At the end of the analysis also a cut on the minimal distance between photon clusters is applied to refine rejection of events with split clusters.

From $1.7 \mathrm{fb}^{-1}$ of data, no events were observed on data in the signal region. Equally, no background events are found in the MC simulation based on twice the data statistics. Hence the KLOE final upper limit is obtained [11]:

$$
\mathrm{BR}\left(\mathrm{K}_{\mathrm{S}} \rightarrow 3 \pi^{0}\right)<2.6 \times 10^{-8} \text { at } 90 \% \text { C.L. }
$$


which improves by almost five times the previous result. This limit can be directly translated into a limit on $\left|\eta_{000}\right|$ :

$$
\left|\eta_{000}\right|=\sqrt{\frac{\tau_{L}}{\tau_{S}} \frac{\mathrm{BR}\left(\mathrm{K}_{\mathrm{S}} \rightarrow 3 \pi^{0}\right)}{\mathrm{BR}\left(\mathrm{K}_{\mathrm{L}} \rightarrow 3 \pi^{0}\right)}}<0.0088
$$

$$
\text { at } 90 \% \text { C.L. . }
$$

\section{CPT and Lorentz symmetry test using entangled neutral kaons}

CPT invariance holds for any realistic Lorentz-invariant quantum field theory. However a very general theoretical possibility for CPT violation is based on spontaneous breaking of Lorentz symmetry, as developed by Kostelecký [12-14], which appears to be compatible with the basic tenets of quantum field theory and retains the property of gauge invariance and renormalizability (Standard Model Extensions - SME). In SME for neutral kaons, CPT violation manifests to lowest order only in the mixing parameter $\delta$, (e.g. vanish at first order in the decay amplitudes), and exhibits a dependence on the 4-momentum of the kaon:

$$
\delta \approx i \sin \phi_{S W} e^{i \phi_{S W}} \gamma_{K}\left(\Delta a_{0}-\overrightarrow{\beta_{K}} \cdot \Delta \vec{a}\right) / \Delta m
$$

where $\gamma_{K}$ and $\overrightarrow{\beta_{K}}$ are the kaon boost factor and velocity in the observer frame, $\phi_{S W}=\arctan (2 \Delta m / \Delta \Gamma)$ is the so called superweak phase, and $\Delta a_{\mu}$ are four CPT- and Lorentz-violating coefficients for the two valence quarks in the kaon.

Following Ref. [13], the time dependence arising from the rotation of the Earth can be explicitly displayed in eq.(11) by choosing a three-dimensional basis $(\hat{X}, \hat{Y}, \hat{Z})$ in a non-rotating frame, with the $\hat{Z}$ axis along the Earth's rotation axis, and a basis $(\hat{x}, \hat{y}, \hat{z})$ for the rotating (laboratory) frame (see Fig.2). The CPT violating parameter $\delta$ may

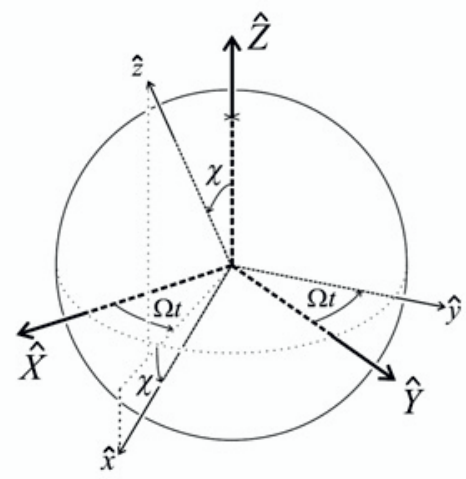

Figure 2. Basis $(\hat{x}, \hat{y}, \hat{z})$ for the rotating frame, and basis $(\hat{X}, \hat{Y}, \hat{Z})$ for the fixed non-rotating frame. The laboratory frame precesses around the Earth's rotation axis $\hat{Z}$ at the sidereal frequency $\Omega$. The angle between $\hat{Z}$ and the positron beam direction $\hat{z}$ defined in the laboratory frame of KLOE is $\chi \simeq 113^{\circ}$. then be expressed as:

$$
\begin{aligned}
\delta\left(\vec{p}, t_{\text {sid }}\right)= & \frac{i \sin \phi_{S W} e^{i \phi_{S W}}}{\Delta m} \gamma_{K}\left\{\Delta a_{0}\right. \\
& +\beta_{K} \Delta a_{Z} \cos \theta \cos \chi \\
& -\beta_{K} \Delta a_{Z} \sin \theta \cos \phi \sin \chi \\
& -\beta_{K} \Delta a_{X} \sin \theta \sin \phi \sin \Omega t_{\text {sid }} \\
& +\beta_{K} \Delta a_{X} \cos \theta \sin \chi \cos \Omega t_{\text {sid }} \\
& +\beta_{K} \Delta a_{X} \sin \theta \cos \phi \cos \chi \cos \Omega t_{\text {sid }} \\
& +\beta_{K} \Delta a_{Y} \cos \theta \sin \chi \sin \Omega t_{\text {sid }} \\
& +\beta_{K} \Delta a_{Y} \sin \theta \cos \phi \cos \chi \sin \Omega t_{\text {sid }} \\
& \left.+\beta_{K} \Delta a_{Y} \sin \theta \sin \phi \cos \Omega t_{\text {sid }}\right\}
\end{aligned}
$$

where $\vec{p}$ is the kaon momentum, $t_{\text {sid }}$ is the sidereal time, $\Omega$ is the Earth's sidereal frequency, $\cos \chi=\hat{z} \cdot \hat{Z} ; \theta$ and $\phi$ are the conventional polar and azimuthal angles of the kaon momentum defined in the laboratory frame about the $\hat{z}$ axis. The sensitivity to the four $\Delta a_{\mu}$ parameters can be very different for fixed target and collider experiments, showing complementary features [13]. At a fixed target experiment usually the kaon momentum direction is fixed, while $|\vec{p}|$ might vary within a certain interval. On the contrary, at a $\phi$-factory kaons are emitted in all directions with the characteristic $p$-wave angular distribution $d N / d \Omega \propto \sin ^{2} \theta$, while $|\vec{p}|$ is almost fixed ${ }^{1}$.

At KLOE the analysis strategy to measure the four $\Delta a_{\mu}$ parameters is based on exploiting the neutral kaon interferometry. In particular when $f_{1}=f_{2}=\pi^{+} \pi^{-}$the corresponding $\eta_{i}$ parameters can be slightly different for the two kaons due to the momentum dependence of the CPT violation effects as come from eq.(12):

$$
\begin{aligned}
& \eta_{1}=\epsilon_{L}+\epsilon^{\prime}=\epsilon-\delta\left(\overrightarrow{p_{1}}, t_{\text {sid }}\right)+\epsilon^{\prime} \\
& \eta_{2}=\epsilon_{L}+\epsilon^{\prime}=\epsilon-\delta\left(\overrightarrow{p_{2}}, t_{\text {sid }}\right)+\epsilon^{\prime},
\end{aligned}
$$

with $\overrightarrow{p_{2}}=\overrightarrow{p_{\phi}}-\overrightarrow{p_{1}}$. The distribution $I\left(f_{1}, f_{2} ; \Delta t\right)$ is extremely sensitive to any deviation from unity of the ratio $\eta_{1} / \eta_{2}$ in the interference region (i.e. $\Delta t \approx 0$ ). Therefore a suitable analysis of the decays $\phi \rightarrow \mathrm{K}_{\mathrm{S}} \mathrm{K}_{\mathrm{L}} \rightarrow \pi^{+} \pi^{-}, \pi^{+} \pi^{-}$ as a function of sidereal time and kaon momenta can provide a measurement of the four parameters $\Delta a_{\mu}$.

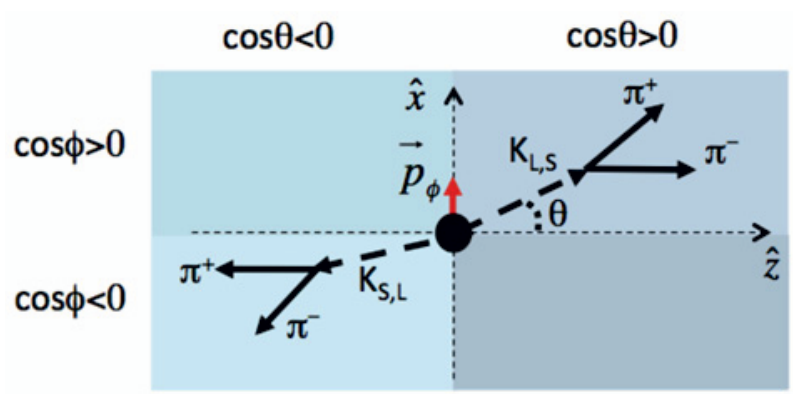

Figure 3. Sketch of the quadrant subdivisions as seen in a top view of the KLOE detector; $\overrightarrow{p_{\phi}}$ is directed along the $\hat{x}$ axis.

\footnotetext{
${ }^{1}$ At DAФNE $|\vec{p}|$ is not fixed because of a small $\phi$ meson momentum $\overrightarrow{p_{\phi}}$ in the laboratory frame $\left(\left|\overrightarrow{p_{\phi}}\right| \simeq 13 \mathrm{MeV} / \mathrm{c}\right)$.
} 


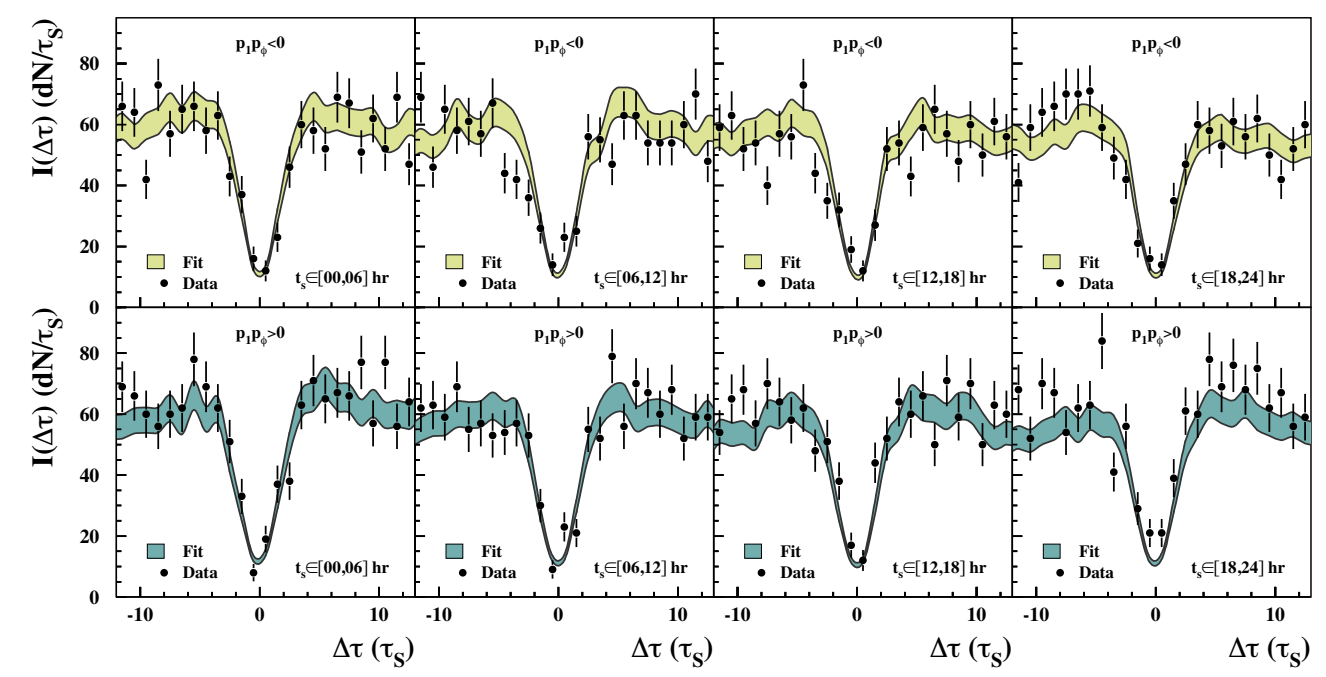

Figure 4. Fit results: the top and bottom plots refer to the two angular selections. Black points are for experimental data while colored bands are the fit output. The error bars on experimental data are purely statistical, while the band represents the contribution to the uncertainty due to MC simulation statistics and efficiency correction.

In the analysis strategy adopted by KLOE the event selection chain is fully symmetric for the two kaon decay vertices in order to minimize any possible systematic effect induced by asymmetric selection criteria. The background contamination in the sample is of the order of $1.7 \%$ and is mainly due to the kaon regeneration on the beam pipe. The signal has a very clean topology, two decay vertices reconstructed from two pairs of tracks, and a global fit using the closed kinematics of the event in the signal hypothesis improves the resolution on the reconstructed time difference $\Delta t$.

The two kaons are distinguished by their emission in the forward $(\cos \theta>0)$ or backward $(\cos \theta<0)$ hemispheres, as sketched in Fig.3. The data sample is divided in two subsets in which the kaons going in the forward direction $(\cos \theta>0)$ are emitted in a quadrant along $(\cos \phi>0)$ or opposite $(\cos \phi<0)$ to the $\phi$ momentum $\overrightarrow{p_{\phi}}$, thus having a higher (or lower) value of $\gamma_{K}$ than the companion kaons emitted in the backward direction (see Fig.3). Moreover the data are divided into four bins of sidereal time. In this way fitting simultaneously the corresponding eight $I\left(\pi^{+} \pi^{-}, \pi^{+} \pi^{-} ; \Delta t\right)$ distributions one is able to observe possible modulation effects induced by the CPT-violating parameter $\delta$ in eq.(12) as a function of sidereal time and kaon momentum. Fig.4.

It is worth noting that the presence of the small momentum $\overrightarrow{p_{\phi}}$ makes $\gamma_{K, 1} \neq \gamma_{K, 2}$ on an event-by-event basis, which is a necessary condition in order to have the $I\left(\pi^{+} \pi^{-}, \pi^{+} \pi^{-} ; \Delta t\right)$ distribution sensitive to the CPT violation effects induced by the $\Delta a_{0}$ parameter.

The fit to the eight $I\left(\pi^{+} \pi^{-}, \pi^{+} \pi^{-} ; \Delta t\right)$ distributions corresponding to $1.7 \mathrm{fb}^{-1}$ of data is shown in T15]:

The results for the four CPT violating parameters are

$$
\begin{aligned}
\Delta a_{0} & =\left(-6.0 \pm 7.7_{\text {stat }} \pm 3.1_{\text {syst }}\right) \times 10^{-18} \mathrm{GeV} \\
\Delta a_{X} & =\left(0.9 \pm 1.5_{\text {stat }} \pm 0.6_{\text {syst }}\right) \times 10^{-18} \mathrm{GeV} \\
\Delta a_{Y} & =\left(-2.0 \pm 1.5_{\text {stat }} \pm 0.5_{\text {syst }}\right) \times 10^{-18} \mathrm{GeV} \\
\Delta a_{Z} & =\left(3.1 \pm 1.7_{\text {stat }} \pm 0.5_{\text {syst }}\right) \times 10^{-18} \mathrm{GeV}
\end{aligned}
$$

with a systematic uncertainty smaller than the corresponding statistical error. These results can be compared to similar ones obtained in the B and D meson systems $[16,17]$, where an accuracy on the $\Delta a_{\mu}^{B, D}$ parameters of $O\left(10^{-13} \mathrm{GeV}\right)$ has been reached.

\section{Perspectives and conclusions}

The KLOE-2 experiment [18] aims to continue and extend the physics program of its predecessor by collecting $O\left(10 \mathrm{fb}^{-1}\right)$ of data at the upgraded DAФNE with an improved KLOE detector.

The KLOE-2 physics program has been described in detail in Ref.[18], including several topics in kaon physics with a special focus on discrete symmetries tests.

The upgrade of the KLOE detector consists of the addition of (i) an inner tracker based on cylindrical GEM technology for the improvement of tracking and decay vertex resolution close to the interaction point (IP), (ii) an $e^{ \pm}$tagging system for the $\gamma \gamma$ physics, and (iii) two calorimeters in the final focusing region to improve acceptance and efficiency for photons coming from the IP and neutral kaon decays inside the detector volume. These new detectors have been built and installed; their commissioning is in an advanced phase, while a new data taking campaign is going to start. In the meanwhile the analysis of the full KLOE data set is being completed. A new preliminary result on the $\mathrm{K}^{+} \rightarrow \pi^{+} \pi^{-} \pi^{+}(\gamma)$ branching ratio has been obtained. A 
new improved upper limit on the branching ratio of the $\mathrm{CP}$-violating decay $\mathrm{K}_{\mathrm{S}} \rightarrow 3 \pi^{0}$ has been measured. At KLOE-2 this analysis will benefit of the presence of the new low $\theta$ calorimeters, and it might be possible to have a first observation of the $\mathrm{K}_{\mathrm{S}} \rightarrow 3 \pi^{0}$ decay with an integrated luminosity of $O\left(10 \mathrm{fb}^{-1}\right)$. A new refined method has been implemented to perform a test of the CPT and Lorentz symmetries using entangled neutral kaons. At KLOE-2 it will benefit of the new inner tracker detector improving the $\Delta t$ resolution, and of the new collision scheme with a doubled $\overrightarrow{p_{\phi}}$ momentum (increasing the sensitivity on the $\Delta a_{0}$ parameter).

\section{Acknowledgements}

The author would like to thank Giorgio Giardina, Giuseppe Mandaglio and all the organizing committee for the pleasant stay in Messina.

\section{References}

[1] M. Adinolfi et al., KLOE coll., Nucl. Instr. and Meth. A 488 (2002) 51.

[2] M. Adinolfi et al., KLOE coll., Nucl. Instr. and Meth. A 482 (2002) 364.

[3] F. Ambrosino, et al., KLOE coll., Phys. Lett. B 666 (2008) 15 .

[4] M. Antonelli, et al., EPJC 69, 399 (2010).

[5] I.H. Chiang, PRD 6(1972), 1254.
[6] T. T. Wu, C. N. Yang, Phys. Rev. Lett. 13, 380 (1964).

[7] A. Di Domenico et al. , Handbook on Neutral Kaon Interferometry at a $\phi$-factory, Frascati Phys. Ser. $\mathbf{4 3}$ (2007).

[8] L. F. Li and L. Wolfenstein, Phys. Rev. D 21, 178 (1980).

[9] L. Maiani, N. Paver $C P$ violation in $K \rightarrow 3 \pi$ decays, and

G. D'Ambrosio, G. Isidori, A. Pugliese $C P$ and $C P T$ measurements at DAФNE, in The second DAPHNE physics handbook, ed. L. Maiani, G. Pancheri, N. Paver, Vol. 1, p.51-62 and 63-95, INFN-LNF, Frascati, 1995.

[10] F. Ambrosino et al., KLOE coll., Phys. Lett. B 619, 61 (2005)

[11] D. Babusci et al., KLOE-2 coll., Phys. Lett. B 723 (2013) 54.

[12] V. A. Kostelecký, Phys. Rev. Lett. 80, 1818 (1998).

[13] V. A. Kostelecký, Phys. Rev. D 61, 016002 (1999).

[14] V. A. Kostelecký, Phys. Rev. D 64, 076001 (2001).

[15] D. Babusci et al., KLOE-2 coll., arXiv: 1312.6818 [hep-ex], submitted to Phys. Lett. B.

[16] B. Aubert, et al., BaBar Coll., Phys. Rev. Lett. 100 (2008) 131802.

[17] J. M. Link, et al., FOCUS Coll., Phys. Lett. B 556 (2003) 7.

[18] G. Amelino-Camelia et al., Eur. Phys. J. C 68, 619 (2010). 